\title{
Dynamic Infrared Thermography of Nanoheaters Embedded in Skin-Equivalent Phantoms
}

\author{
K. A. López-Varela $\mathbb{D}^{1},{ }^{1}$ N. Cayetano-Castro, ${ }^{2}$ E. S. Kolosovas-Machuca, ${ }^{3}$ F. J. González $\mathbb{D}{ }^{3}$, \\ F. S. Chiwo, ${ }^{3}$ and J. L. Rodríguez-López $\mathbb{1}^{1}$ \\ ${ }^{1}$ División de Materiales Avanzados, Instituto Potosino de Investigación Científica y Tecnológica, A.C., \\ Camino Presa San José 2055, Lomas $4^{a}$ Secc., 78216 San Luis Potosí, SLP, Mexico \\ ${ }^{2}$ Nanoscience, Micro and Nanotechnology Center, National Polytechnic Institute, Av. Luis Enrique Erro s/n, \\ Zacatenco, 07738 Mexico City, Mexico \\ ${ }^{3}$ Coordinación para la Innovación y la Aplicación de la Ciencia y la Tecnología, \\ Universidad Autónoma de San Luis Potosí, San Luis Potosí, SLP, Mexico
}

Correspondence should be addressed to K. A. López-Varela; karla.lopez@ipicyt.edu.mx and J. L. Rodríguez-López; jlrdz@ipicyt.edu.mx

Received 13 July 2017; Revised 1 November 2017; Accepted 5 December 2017; Published 14 January 2018

Academic Editor: Leszek A. Dobrzański

Copyright (C) 2018 K. A. López-Varela et al. This is an open access article distributed under the Creative Commons Attribution License, which permits unrestricted use, distribution, and reproduction in any medium, provided the original work is properly cited.

\begin{abstract}
Nanoheaters are promising tools for localized photothermal therapy (PTT) of malignant cells. The anisotropic AuNPs present tunable surface plasmon resonances (SPR) with ideal NIR optical response to be applied as theranostic agents. To this purpose, nanoparticles with branches are suitable because of the electromagnetic field concentrated at their vertices. We standardized a protocol to synthesize multibranched gold nanoparticles (MB-AuNPs) by the seed-growth method and found a size-seed dependence tunability on the hierarchy of branching. Once the optical response is evaluated, we tested the temporal stability as nanoheaters of the MB-AuNPs immersed in skin-equivalent phantoms by dynamic infrared thermography (DIRT). The most suited sample presents a concentration of $5.2 \times 10^{8} \mathrm{MB}$-AuNPs $/ \mathrm{mL}$ showing good thermal stability with $\Delta T=4.5^{\circ} \mathrm{C}$, during $3 \mathrm{cycles}$ of $10 \mathrm{~min}$ at $785 \mathrm{~nm}$ laser irradiation with power of $0.15 \mathrm{~W}$. According to these results, the MB-AuNPs are suitable nanoheaters to be tested for PTT in more complex models.
\end{abstract}

\section{Introduction}

The last twenty years' research reports on nanostructured materials clearly indicate their potential to develop new technologies for different specialized areas. The extensive investigation work in shape controlled synthesis of metal nanoparticles (NPs) has allowed the achievement of structures with complicated geometric forms and the use of nontoxic chemicals for their synthesis. Gold plays a particular and special role in this area of size and shape controlled synthesis, and because of their properties, those NPs are studied for technological applications in fields such as renewable energies, catalysis, medicine, and photonics. Because of the optical and low-reactive properties of gold nanoparticles (AuNPs), they have been considered for medical applications as theranostic agents, which means that they can be simultaneously used for drug delivery [1], medical imaging [2-5], localized photothermal therapy (PTT) [6], and biological sensing $[7,8]$.

Nowadays, for some specific theranostic applications AuNPs are designed with anisotropic shapes, characteristic that tunes their surface plasmon resonance (SPR) from the visible to the near (NIR) and middle infrared (Mid-IR) regions of the spectra, also presenting local concentration of electromagnetic fields on the vertices [9]. The most common anisotropic AuNPs studied and used have been the gold nanorods (AuNRs) and stellated or spiky AuNPs, here termed multibranched gold nanoparticles (MB-AuNPs). The fact that NIR absorbance of MB-AuNPs fits the so-called therapeutic 
window present in the human tissue (700-1200 nm) [10], a spectral region where maximum penetration of light occurs due to the minimal absorption of blood components, such as hemoglobin and water [11], is important for these applications.

With the goal to apply these gold nanoheaters on the PTT of cancer (localized heating for killing cancer cells) [12], we have to understand their thermal response under controlled conditions. Thermal response has been evaluated by dynamic thermography technique, as infrared imaging tool for realtime recording of the temperature increases $(\Delta T)$. Since the evaluation of nanosystems in real tissue samples presents many out-of-control factors, such as the complexity in the formation of protein corona on the nanoparticles and their interaction with the physiological cellular environment [13, 14], and besides the lack of the reproducibility of results due to the difficulty in finding identical specimens, we decided the use of gel phantoms as skin-equivalent model [12, 1517] which emulates the dielectric properties of the human body surface. Previous reports had evaluated the thermal response of AuNRs [18] in agar phantoms with intralipid ${ }^{\circledR}$ (fat emulsion) as scattered agent [19] and suggested to be effective for tumor irradiation of $10 \mathrm{~mm}$ depth from the illuminated tissue surface. We use for the accurate analysis of MBAuNPs nanoheaters an optimal agar phantom model, with permittivity close to that of the human skin in the $60 \mathrm{GHz}$ band [20]. The skin-equivalent phantom uses polyethylene powder (PEP) to decrease the real and imaginary parts of the permittivity.

In this report we use the organic molecule HEPES, 2-[4(2-hydroxyethyl)-1-piperazinyl] ethanesulfonic acid, as shape directing agent. HEPES is one of the good buffers used in cell culture [21] and is also used as reducing and shape directing agent $[22,23]$ in the synthesis of nanoparticles by wet-chemical [24-26] and hydrothermal methods [27]. The shape promoted by HEPES in nanoparticle formation is mostly multibranched, varying from few to several peaks.

Previously reported seeded-growth synthesis protocol, in which the HEPES act as directing agent and hydroxylamine $\left(\mathrm{NH}_{2} \mathrm{OH} \cdot \mathrm{HCl}\right)$ [28-30], is used as reducing agent [31] over the seeds surface; the hydroxylamine has the role of avoiding new nucleation events during the peak growth stage. It has been also reported that hydroxylamine influences or promotes the growth of stellated gold nanoparticles [32]. Thus, we start from this method that involves two molecules, both with capping and direct-shaping properties. The function of the HEPES and hydroxylamine during the growing stage of peaks has been determined by our experimental design on concentrations of the reactants. The seed-growth method allows obtaining good monodispersity in the final product shape and size. We also had found a size-seed dependence tunability on the hierarchy of branching.

In this work, we evaluated the optical response of $\mathrm{MB}$ AuNPs by UV-Vis spectroscopy, and their morphology by electronic microscopy (SEM and HRTEM). Particle density was determined by inducted coupled plasma spectrometry (ICP), and the temporal stability as nanoheaters was tested in skin-equivalent phantoms by dynamic infrared thermography (DIRT).

\section{Materials and Methods}

Deionized water was used for all experiments. The reagents used for NPs synthesis were obtained from Sigma-Aldrich: $\mathrm{HAuCl}_{4} \cdot 3 \mathrm{H}_{2} \mathrm{O}, \mathrm{HEPES}, \mathrm{NH}_{2} \mathrm{OH} \cdot \mathrm{HCl}$, and sodium citrate. For the preparation of skin-equivalent-phantom, polyethylene powder (PEP) was obtained from Baker, Agar from Sigma-Aldrich, and TX-151 from Oil Center, and all reagents were used as received without further treatment or purification. All glassware was cleaned with aqua regia $\left(\mathrm{HCl}: \mathrm{HNO}_{3}\right.$, $3: 1$ ) and rinsed with plenty of deionized water. The samples were characterized with UV-Vis absorption spectroscopy, acquired on a Cary $60 \mathrm{UV}-\mathrm{Vis}-\mathrm{NIR}$ spectrophotometer at room temperature. ICP elemental quantification was performed on Variant 730-ES spectrometer. The electron micrographs were acquired in a transmission electron microscope JEOL 2100, and scanning electron micrographs in a FEIHelios Nanolab. The X-ray diffractograms were obtained in a Bruker DX-8 system at CuK $\alpha$ source of wavelength $1.54056 \AA$. The dynamic thermographs were taken at a distance of $0.3 \mathrm{~m}$ from the skin-equivalent phantom [15, 33], using a high-resolution infrared camera with thermal sensitivity better than $40 \mathrm{mK}$ and a $480 \times 360$ focal plane array of VOx microbolometers (FLIR T600, FLIR Systems Inc., Wilsonville, OR). The phantoms were evaluated with a $785 \mathrm{~nm}$ laser diode with a spot size of $3.5 \mathrm{~mm}$ and optical power of $0.15 \mathrm{~W}$. Temperature measurements of the skin-like phantom were taken with and without MB-AuNPs. Data was analyzed with FLIR-IR Research software.

2.1. Synthesis of Multibranched Gold Nanoparticles (MBAuNPs) Using a Seed-Growth Method. The 16 and 18 gold nanospheres (AuNSs) used as seeds were prepared by a reverse-modified Turkevich method [34] by citrate reduction of gold salt. Shortly, in a three-neck round-bottom flask with a condenser mounted, a citrate solution was brought to boiling by a heating mantle for 5 mins; then a gold solution of initial concentration of $25 \mathrm{mM}$ was added, and after a light red ruby color appeared the system was left to react for half hour. For the synthesis of $16 \mathrm{~nm}$ AuNSs, the total concentration of sodium citrate and gold salt was $1.8 \mathrm{mM}$ and $0.16 \mathrm{mM}$, respectively; and for $18 \mathrm{~nm}$ AuNSs were $0.75 \mathrm{mM}$ and $0.25 \mathrm{mM}$. After the reaction was completed, the seeds solution was left to cool down at ambient temperature and used without modifications.

For the MB-AuNPs synthesis we used three concentrations of HEPES buffer [25, 50, $75 \mathrm{mM}$ ] as solution. Briefly, under slowly stirring within an ice bath $\left(\sim 4^{\circ} \mathrm{C}\right)$ to $12.5 \mathrm{~mL}$ of HEPES $50 \mu \mathrm{L}$ of AuNSs (16 or $18 \mathrm{~nm}$ ) was added, as well as 30,60 , or $120 \mu \mathrm{L}$ of hydroxylamine [0.1 M], and finally the dropwise addition of gold salt solution with rate of $0.375 \mathrm{~mL} / \mathrm{min}$ to a final concentration of 0.07 or $0.13 \mathrm{mM}$. For the reaction to be complete it is necessary to leave the sample to rest $8 \mathrm{~h}$ in the fridge. The samples were washed three times by centrifugation and a drop of the concentrated colloid was deposited on an aluminum pin for SEM and on a lacey carbon copper grid for HRTEM. For UV-Vis measurements, the samples were analyzed as synthesized. The samples for ICP quantification were centrifuged and the NPs concentrated was dissolved in aqua regia. 
2.2. Fabrication of Skin-Equivalent Phantom. For the fabrication of the control phantom a previously reported protocol was used, and the reagents concentration was maintained [20]. The procedure is as follows: a Buchner flask with deionized water and agar was heated until $\sim 80^{\circ} \mathrm{C}$ under slow magnetic stirring and vacuum. Once the agar was dissolved and the boiling point was reached, the vacuum and heating were turned off, the TX-151 was added and mixed gently with a stirring rod until its complete incorporation, and then the PEP was added to decrease the real and imaginary parts of the permittivity. After complete PEP incorporation the vacuum was turned on for a moment to avoid bubbles in the final phantom. Finally, the mix was placed into a petri box $(\varnothing=55 \mathrm{~mm})$ and left to solidify at ambient temperature. When phantoms with nanoparticles were prepared, a $1 \mathrm{x}$ concentrated of prewashed MB-AuNPs colloid was added and completely incorporated before TX-151 addition. For comparison, other phantoms with AuNSs were prepared, at the same NPs concentration of MB-AuNPs.

\section{Results}

3.1. Multibranched Gold Nanoparticles (MB-AuNPs). In order to prepare monodisperse MB-AuNPs, presynthesized gold single-crystalline and multitwinned nanoparticles are used as seeds for the growth of peaks $[28,29]$, which grow along preferential crystallographic directions of the metallic core [35]. According to previous reports, the capping agents [36] have preferential adsorption by specific crystalline faces of the metal seeds [37]; and this promotes an accelerated growth rate along specific crystallographic directions [7, 32, 38-40].

The AuNSs, obtained by the reverse Turkevich method, were monodispersed in size (see Figures 1 and SI-1); by ICP spectroscopy was determined the concentration of $2.39 \times 10^{11}$ and $3.66 \times 10^{11} \mathrm{NPs} / \mathrm{mL}$ for 16 and $18 \mathrm{~nm}$ AuNSs, respectively. We will see later that the structural characteristics of the AuNSs will influence the growth process of peaks; as can be seen in Figure 1(a), the 16-AuNSs present more symmetrical shape and monocrystalline structure, being different from 18 AuNSs samples (Figure 1(a)) that show multitwinned structure and nonspherical multifaceted shapes. During the stage of peaks formation, the promotion of second-order branching (Figure 3) is up to the seed structure [41] and the stabilizer agent protection [7] at the nanoparticle surface, observed in the MB-AuNPs synthesized from $18 \mathrm{~nm}$ nanospheres (18 MBAuNPs), with $0.13 \mathrm{mM}$ of gold salt. Also, we observed that the growth mechanism of the MB-AuNPs is influenced by the chemical characteristics of the reagents present in the growth solution and by its molar ratio with respect to gold ions.

Therefore, both hydroxylamine and HEPES are dual agents, with reducing and shape capabilities. Manipulation of the role played by each reactive is made through experimental conditions such as temperature (e.g., to slow the HEPES reducing action) and concentration of hydroxylamine (using the necessary to reduce the gold ions). Within the molar rates used in this work, the hydroxylamine governs the reaction kinetics and the final shape of the peaks and the HEPES modulate the peaks growth. A simple way to know if the HEPES has been or not contributing as reducing agent is by means of UV-Vis spectroscopy (see Figure SI-2), since the nitrogen species from degraded HEPES can be detected by its absorption at $\sim 346 \mathrm{~nm}$ [22]. It is important to mention that, in the reaction where no hydroxylamine is used, the color changes of the colloid take place slowly, evidencing that HEPES is a slow reductor. Increasing the concentration of HEPES (from 25 to $50 \mathrm{mM}$ ), the growth of narrow peaks is promoted while the cores remain small. Then the addition of hydroxylamine is necessary to modulate the kinetics without excess of HEPES and will help to obtain better definition on the peaks and a monodisperse size and shape sample (see Figure SI-3). From the mechanism reduction of $\mathrm{Au}^{3+}$ by hydroxylamine proposed by Minati et al. [42], the molar ratio of $\mathrm{Au}^{3+}: \mathrm{NH}_{2} \mathrm{OH}(1: 3)$ was used to synthesize the sample with $16 \mathrm{~nm}$ nanospheres (16 MB-AuNPs), in order to avoid the intervention of HEPES reducing action (see Figure SI-2). We observed that, when hydroxylamine is present and increases its molar rate, the change of colors gets faster indicating the necessary use of a mild reducing agent (hydroxylamine) simultaneously with a directing shape agent (HEPES) for better anisotropic growth. We also have determined that an excess of hydroxylamine promotes the second-order branching in $18 \mathrm{MB}$-AuNPs, which was synthesized with molar rate of $\mathrm{Au}^{3+}: \mathrm{NH}_{2} \mathrm{OH}$ of 1:6 (see Figure SI-4).

It had been reported that the size of the NPs used as seeds influences the shape of final products [30, 43]. In our results we observed that from 16-AuNSs, MB-AuNPs of $80 \mathrm{~nm}$ of diameter can be obtained, synthesized with $0.07 \mathrm{mM}$ of gold salt (16 MB-AuNPs, see Figure SI-5), and the colloid presents a maximum absorbance at $728 \mathrm{~nm}$ (Figure 2). On the other hand, dendritic growth is only perceptible in $18 \mathrm{MB}$-AuNPs (Figure 3), even if the concentration of gold ions is increased in the samples synthesized from 16-AuNSs (see Figure SI-6). The increase in gold ions leads to bigger cores and smaller length of peaks, due to an addition of gold atoms between peaks for minimization of energy, instead of the growth of longer peaks $[44,45]$.

At this stage we have shown that both sets of samples (16 and $18 \mathrm{MB}$-AuNPs) present good optical response for being used as nanoheaters. The next criteria for choosing a sample is the critical size for being used in an in vitro or in vivo environment. Thus, we decided the use of $16 \mathrm{MB}$ AuNPs for being tested as nanoheaters; however, the optical response of $18 \mathrm{MB}$-AuNPs in the $830 \mathrm{~nm}$ (Figure 3) is suitable for applications in nanostructured system for SERS [46].

3.2. Thermal Response of MB-AuNPs. Infrared (IR) imaging is based on the fact that any object at a temperature above absolute zero $\left(-273^{\circ} \mathrm{C}\right)$ will emit IR radiation, even if only weakly. The human body has a low thermal emittance, radiating in a wavelength range that starts at around $3 \mu \mathrm{m}$ and peaks in the vicinity of $10 \mu \mathrm{m}$ and trails off from this point into the extreme IR and, negligibly, beyond it. The emissivity of human skin has a constant value between wavelengths of 2 and $14 \mu \mathrm{m}$ of $0.98 \pm 0.01$ for black skin and $0.97 \pm 0.02$ for white skin [47]; thus, human skin has a known and almost invariant emissivity in this wavelength region that makes IR imaging an ideal procedure to evaluate surface temperature of 


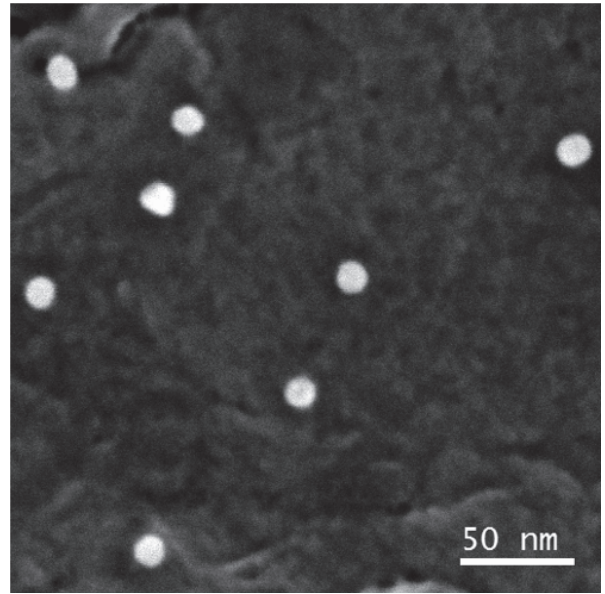

(a)

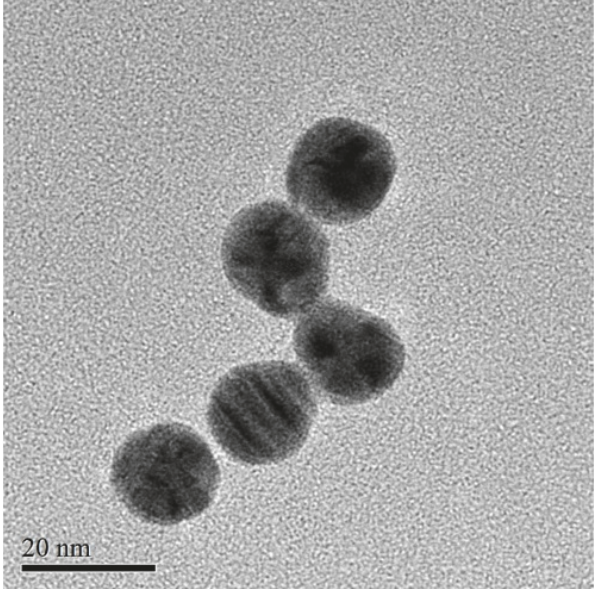

(b)

FIGURE 1: (a) SEM micrograph of $16 \pm 1.69 \mathrm{~nm}$ of spheres gold nanoparticles (16-AuNSs). (b) TEM micrograph of $18.18 \pm 2.33 \mathrm{~nm}$ sphere gold nanoparticles (18-AuNSs) used as seeds.

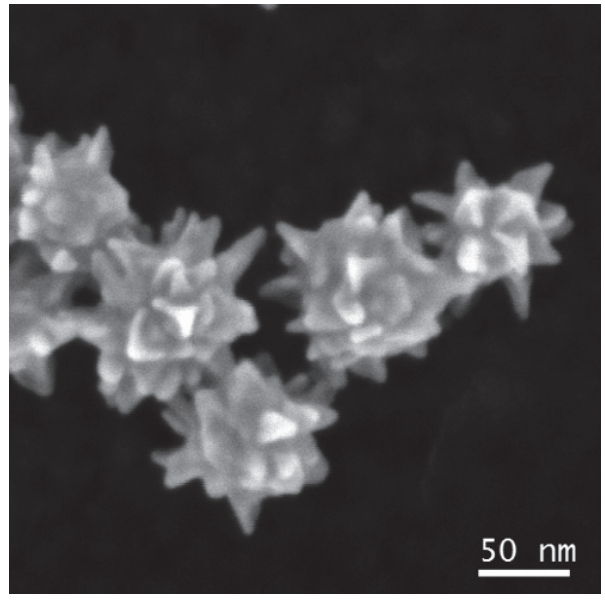

(a)

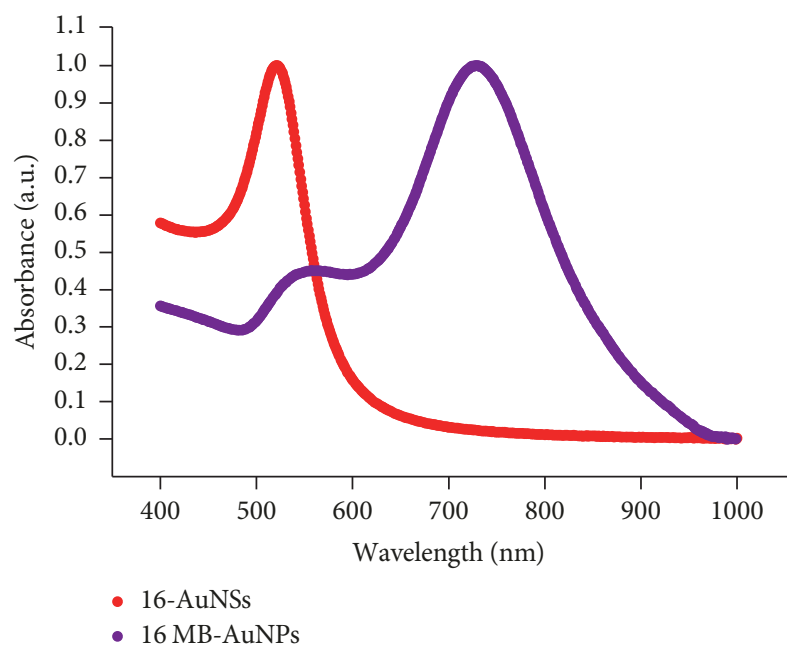

(b)

FiguRE 2: (a) SEM micrograph of 16 MB-AuNPs synthetized with 16-AuNSs. (b) UV-Vis spectra of 16-AuNSs and 16 MB-AuNPs with a band centered at $520 \mathrm{~nm}$ and $729 \mathrm{~nm}$, respectively.

the human body $[48,49]$. Because of the above arguments, the skin-equivalent phantoms with $0.62 \pm 0.01$ reported emittance [17] is a well suited model for IR thermal evaluation.

The thermal response of 16-AuNSs and $16 \mathrm{MB}$-AuNPs was evaluated inside the skin-equivalent phantoms by dynamic thermography. The samples were irradiated at $0.15 \mathrm{~W}$ power laser by 3 cycles of 10 mins and recorded with an IR camera, and the thermographs shown at Figure 4 were extracted at $555 \mathrm{~s}$ using the FLIR-RIR software, which allows the analysis of single points, line profiles, and averages of circle/rectangular areas. The plot in Figure 4(a) shows data from a line profile analyzed at different times, and it clearly can be observed that not only the incident laser area has been heated but also there is a radial propagation by diffusion of the generated heat; thus there is energy transmitted to the adjacent regions due to the immersed NPs. This effect is also observed in the control phantom analysis, but over a smaller area and with more uniform temperature as can be seen in Figure SI-7. Figure 4(b) reports the information on how fast the system absorbs the energy, achieving constant temperature at early irradiation times (60 s). This was also performed for evidence the continuity of the thermal response of immersed $16 \mathrm{MB}$ AuNPs in the phantom as a function of time.

The plot in Figure 5 corresponds to a dynamic thermal analysis, and from it we can appreciate the difference in the temperature reached by the three phantoms: the largest 


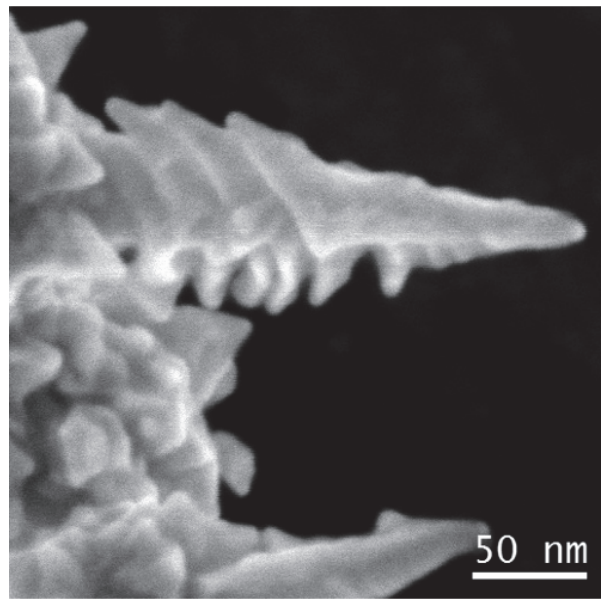

(a)

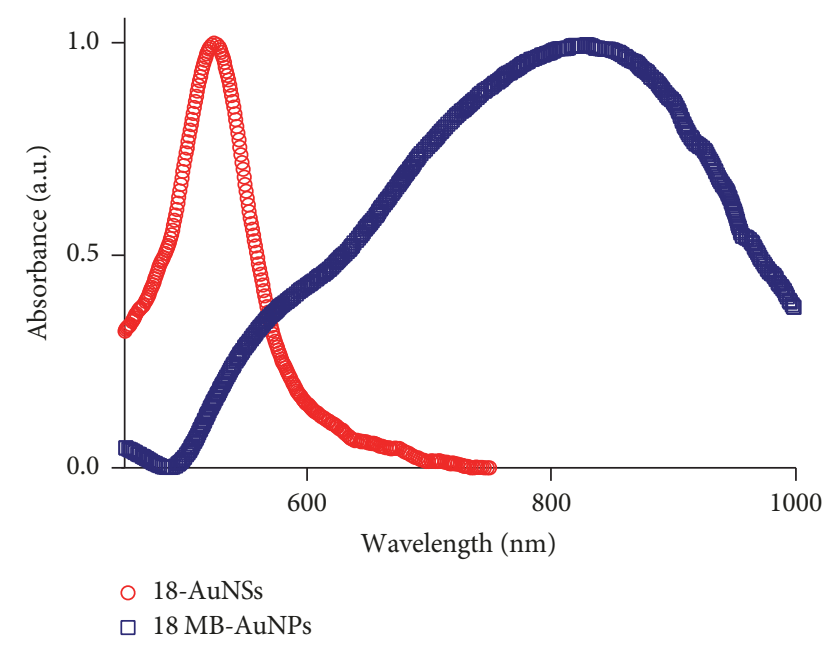

(b)

FIGURE 3: SEM micrographs (a) of 18 MB-AuNPs with second order branches and UV-Vis spectra (b) of 18-AuNSs and 18 MB-AuNPs; they have a band centered at $524 \mathrm{~nm}$ and $830 \mathrm{~nm}$, respectively.
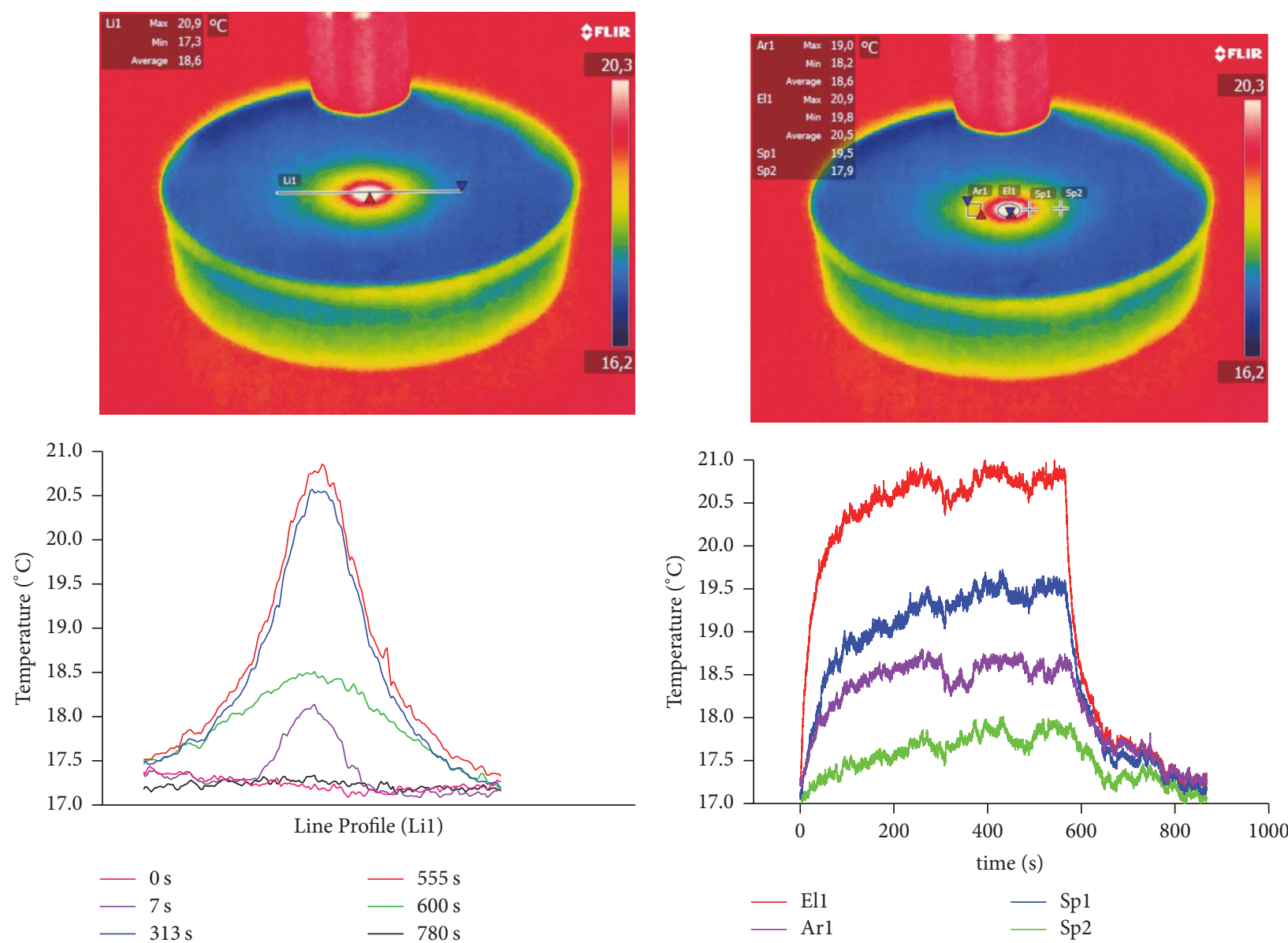

(a)

(b)

FIGURE 4: The thermography corresponds at the $555 \mathrm{~s}$ of laser irradiation. (a) Temperature profiles of the heating area at different times. The profile analyzed is designed as Line 1 (Lil) in the thermography. (b) Temporal analysis of temperature variations of different points and regions marked in the thermography as area (Ar1), elipsoide (El1), and singles points (Sp1, Sp2). 


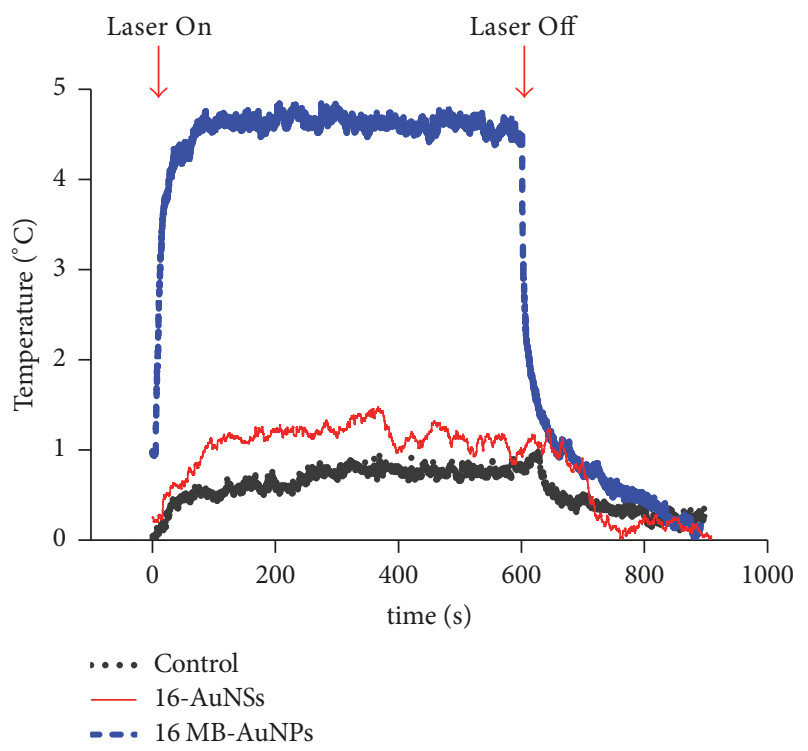

FIGURE 5: Thermal response of skin-equivalent phantoms with 16AuNSs, 16 MB-AuNPs, and control.

increase of localized temperature is measured in the sample prepared with $16 \mathrm{MB}-\mathrm{AuNPs}$, compared to the phantom with 16-AuNSs and the one without the addition of NPs, used as a control.

A red-shift of the LSPR is present for anisotropic MBAuNPs compared with spheres (see Figure 1), and a substantial increase in the heating efficiency with respect the sphere is expected. In our results, the maximum temperature increment in 16 MB-AuNPs phantom was achieved in $60 \mathrm{~s}$ and maintained during all the laser irradiation. This temperature increment is $\Delta T=4.5^{\circ} \mathrm{C}$ and when the light source is turned off, a fast decay is observed. The comparison between the AuNSs and MB-AuNPs at the same concentration shows the evident increase of thermal response due to the anisotropic shape of MB-AuNPs and the localized SPR's synergy, because the MB-AuNPs absorbs closer to the wavelength of laser irradiation. In the case of the phantom with 16-AuNSs, the increment in temperature can be described for the excitation of hot-spots created in the interaction among spherical NPs $[50,51]$. The fact that there were not physical changes observed in the phantoms under laser irradiation, as leaking or deformation, even after the three cycles evaluation is important to mention.

\section{Discussion}

We have shown that the control on the width, the length, the number of peaks, and the degree of dendritic growth gives rise to an easy tuning of the plasmon resonance spectra [6] and their corresponding higher transduction of light into heat, and therefore gold nanoheaters are good candidates for IR absorption for efficient photothermal therapy of malignant cancer cells.

The structural characteristics of the AuNSs influenced the degree of ramification of the nanoparticle tips, resulting in second-order branches for MB-AuNPs synthesized from 18AuNSs, which present crystalline defects promoting directed anisotropy. From 16-AuNSs we could obtain monodisperse samples. After analyzing the impact of HEPES and hydroxylamine on the final tips shape, we estimated that the optimal molar ratio of hydroxylamine: Au ions is $3: 1$, where this amount is enough to avoid the intervention of HEPES in the reduction. Even if an excess of hydroxylamine may result in a better definition of tips, it can also cause the agglomeration of MB-AuNPs, due to the resulting high ionic force of the solution, that could not be used for clinical applications, and the same argument is valid for higher concentration of HEPES $(75 \mathrm{mM})$. On the other side, the increase on the molar concentration of $\mathrm{Au}$ ions (and necessary hydroxylamine) leads to bigger cores and smaller length of tips; thus it shifts the SPR to lower wavelengths making them not practical for PTT.

Regarding the dynamic thermography results for $16 \mathrm{MB}$ AuNPs, we found that not only the incident laser area is being heated, but also there is a radial propagation of the released heat, which means there is transmission of energy around the adjacent region of the spot due to the immersed NPs. Therefore, the addition of MB-AuNPs results in the production of more efficient centers for scattering and reemission of energy.

The temperature increment achieved by the nanoheaters in this work $\left(\Delta T \sim 5^{\circ} \mathrm{C}\right)$ is good enough for effective and minimally harmful gold nanoparticles based photothermal therapy techniques [52]. This temperature increment from multibranched gold nanoparticles immersed in a phantom gel (as a model approach for the real environment in bioapplications) satisfies biomedical requirement for the treatment of superficial diseases (mycoses, fungal infections, and many different types of skin cancer) [53].

\section{Conclusions}

A synthesis method that uses nonaggressive chemicals for obtaining multibranched gold nanoparticles and secondary growth branching by tuning the seed size is presented. Analysis of the UV-Vis spectra and SEM micrographs allows us to understand the role of each reactant in the final shape of the nanoparticles. The HEPES molecule mainly grow tips from seeds with a random order; and the hydroxylamine can act as a directing shape agent, but this role only follows after its reduction role has been finished in the reaction. A faster depletion of the reactants (by higher concentration of hydroxylamine) during the grow reaction promotes the growth of larger tips with second-order branches.

We have determined that the synthesized multibranched gold nanoparticles increase the temperature in a localized area irradiated with a $785 \mathrm{~nm}$ laser. The temperature increment recorded was $\Delta T=4.5^{\circ} \mathrm{C}$. The $16 \mathrm{MB}$-AuNPs embedded in the phantom gel is a system that rapidly absorbs the proper incident energy, achieving a plateau of constant temperature within $60 \mathrm{~s}$ which is recovered during at least three different cycles. This evaluation indicates MB-AuNPs as an interesting system for being tested as nanoheater in biological models. Furthermore, the evaluation of their photothermal behavior in cultured cells is necessary for their direct application in 
medicine. We also propose to carry on the test with an irradiance lower than the ANSI regulation. Due to the experimental conditions reached in our phantoms, we expect that the transduction of light into thermal energy will be more effective, due to the limitation of MB-AuNPs reshaping.

\section{Conflicts of Interest}

The authors declare that they have no conflicts of interest.

\section{Acknowledgments}

The authors acknowledge Ph.D. CONACYT scholarship number 375630 and institutional support from IPICYT. The authors acknowledge Dra. Gladys Judith Labrada-Delgado for SEM and Dr. Hector Gabriel Silva-Pereyra for HRTEM analysis, as well as the infrastructure of the National Laboratory for Nanoscience and Nanotechnology (LINAN) at IPICYT. This work has been supported by CONACYTMexico Grants 106437 and 216315. F. J. González would like to acknowledge support from Project 32 of "Centro Mexicano de Innovación en Energía Solar" and by the National Laboratory Program from CONACYT through the Terahertz Science and Technology National Lab (LANCYTT).

\section{Supplementary Materials}

Figure SI-1. Histograms of diameters and frequency distribution data of populations of (a) 200 AuNSs for 16-AuNSs and (b) 100 for 18-AuNSs. Figure SI-2. The increase of the molar concentration of HEPES promotes strong anisotropic formation of the MB-AuNPs. Figure SI-3. Scanning electron microscopy micrograph of $16 \mathrm{MB}$-AuNPs; the monodispersity and nonagglomeration of the NPs can be observed. Figure SI-4. Diagram of the concentrations of reactants used for the samples analyzed. Figure SI-5. Histograms of diameters and frequency distribution data of populations of $5016 \mathrm{MB}$-AuNPs. Figure SI-6. SEM micrographs of MBAuNPs synthesized in a medium of HEPES [50 mM], with a molar ratio Au3+/NH2OH of $1: 3$, with (a) 16-AuNSs and (b)(c) 18-AuNSs. Figure SI-7. Thermography of skin-equivalent phantom used as control. (Supplementary Materials)

\section{References}

[1] T. Lajunen, L. Viitala, L.-S. Kontturi et al., "Light induced cytosolic drug delivery from liposomes with gold nanoparticles," Journal of Controlled Release, vol. 203, pp. 85-98, 2015.

[2] A. Guerrero-Martínez, S. Barbosa, I. Pastoriza-Santos, and L. M. Liz-Marzán, "Nanostars shine bright for you: colloidal synthesis, properties and applications of branched metallic nanoparticles," Current Opinion in Colloid \& Interface Science, vol. 16, no. 2, pp. 118-127, 2011.

[3] Z. Fan and H. Zhang, "Template synthesis of noble metal nanocrystals with unusual crystal structures and their catalytic applications," Accounts of Chemical Research, vol. 49, no. 12, pp. 2841-2850, 2016

[4] W. Park, S. Cho, X. Huang, A. C. Larson, and D. Kim, "Branched Gold Nanoparticle Coating of Clostridium novyi-NT Spores for
CT-Guided Intratumoral Injection," Small, vol. 13, no. 5, Article ID 1602722, 2017.

[5] Y. Ponce De León, J. L. Pichardo-Molina, N. Alcalá Ochoa, and D. Luna-Moreno, "Contrast enhancement of optical coherence tomography images using branched gold nanoparticles," Journal of Nanomaterials, vol. 2012, Article ID 571015, 9 pages, 2012.

[6] A. Espinosa, A. K. A. Silva, A. Sánchez-Iglesias et al., "Cancer cell internalization of gold nanostars impacts their photothermal efficiency in vitro and in vivo: toward a plasmonic thermal fingerprint in tumoral environment," Advanced Healthcare Materials, vol. 5, no. 9, pp. 1040-1048, 2016.

[7] J. P. Xie, Q. B. Zhang, J. Y. Lee, and D. I. C. Wang, "The synthesis of SERS-active gold nanoflower tags for in vivo applications," ACS Nano, vol. 2, no. 12, pp. 2473-2480, 2008.

[8] M. Aioub and M. A. El-Sayed, "A Real-time surface enhanced raman spectroscopy study of plasmonic photothermal cell death using targeted gold nanoparticles," Journal of the American Chemical Society, vol. 138, no. 4, pp. 1258-1264, 2016.

[9] X.-L. Liu, J.-H. Wang, S. Liang et al., "Tuning plasmon resonance of gold nanostars for enhancements of nonlinear optical response and raman scattering," The Journal of Physical Chemistry C, vol. 118, no. 18, pp. 9659-9664, 2014.

[10] F. J. González, "Noninvasive detection of filaggrin molecules by raman spectroscopy," in Filaggrin, pp. 93-101, Springer Berlin Heidelberg, Berlin, Germany, 2014.

[11] H. Berlien and G. J. Müller, Applied Laser Medicine, Springer, Berlin, Heidelberg, Germany, 2003.

[12] R. Ahmad, J. Fu, N. He, and S. Li, "Advanced Gold Nanomaterials for Photothermal Therapy of Cancer," Journal of Nanoscience and Nanotechnology, vol. 16, no. 1, pp. 67-80, 2016.

[13] P. Foroozandeh and A. A. Aziz, "Merging worlds of nanomaterials and biological environment: factors governing protein corona formation on nanoparticles and its biological consequences," Nanoscale Research Letters, vol. 10, no. 1, p. 221, 2015.

[14] D. Walczyk, F. B. Bombelli, M. P. Monopoli, I. Lynch, and K. A. Dawson, "What the cell "sees" in bionanoscience," Journal of the American Chemical Society, vol. 132, no. 16, pp. 5761-5768, 2010.

[15] G. P. Mazzara, R. W. Briggs, Z. Wu, and B. G. Steinbach, "Use of a modified polysaccharide gel in developing a realistic breast phantom for MRI," Magnetic Resonance Imaging, vol. 14, no. 6, pp. 639-648, 1996.

[16] M. N. Iizuka, M. D. Sherar, and I. A. Vitkin, "Optical phantom materials for near infrared laser photocoagulation studies," Lasers in Surgery and Medicine, vol. 25, no. 2, pp. 159-169, 1999, http://www.ncbi.nlm.nih.gov/pubmed/10455223.

[17] Y. Okano, K. Ito, I. Ida, and M. Takahashi, “The SAR evaluation method by a combination of thermographic experiments and biological," IEEE Trans. Microw. Theory Tech, vol. 48, pp. 20942103, 2000.

[18] N. R. Jana, L. Gearheart, and C. J. Murphy, "Wet chemical synthesis of high aspect ratio cylindrical gold nanorods," The Journal of Physical Chemistry B, vol. 105, no. 19, pp. 4065-4067, 2001.

[19] C. L. Didychuk, P. Ephrat, A. Chamson-Reig, S. L. Jacques, and J. J. L. Carson, "Depth of photothermal conversion of gold nanorods embedded in a tissue-like phantom," Nanotechnology, vol. 20, no. 19, Article ID 195102, 2009.

[20] N. Chahat, M. Zhadobov, S. Alekseev, and R. Sauleau, "Human skin-equivalent phantom for on-body antenna measurements in $60 \mathrm{GHz}$ band," IEEE Electronics Letters, vol. 48, no. 2, pp. 6768, 2012. 
[21] N. E. Good and S. Izawa, "Hydrogen Ion Buffers," Methods in Enzymology, vol. 24, no. C, pp. 53-68, 1972.

[22] A. Habib, M. Tabata, and Y. G. Wu, "Formation of Gold Nanoparticles by Good's Buffers," Bulletin of the Chemical Society of Japan, vol. 78, no. 2, pp. 262-269, 2005.

[23] J. Xie, J. Y. Lee, and D. I. C. Wang, "Seedless, surfactantless, highyield synthesis of branched gold nanocrystals in HEPES buffer solution," Chemistry of Materials, vol. 19, no. 11, pp. 2823-2830, 2007.

[24] R. Chen, J. Wu, H. Li, G. Cheng, Z. Lu, and C.-M. Che, "Fabrication of gold nanoparticles with different morphologies in HEPES buffer," Rare Metals, vol. 29, no. 2, pp. 180-186, 2010.

[25] Y. Bao, H.-C. Yeh, C. Zhong et al., "Formation and stabilization of fluorescent gold nanoclusters using small molecules," The Journal of Physical Chemistry C, vol. 114, no. 38, pp. 15879-15882, 2010.

[26] S. Chen, Q. Lei, W.-X. Qiu et al., "Mitochondria-targeting "Nanoheater" for enhanced photothermal/chemo-therapy," Biomaterials, vol. 117, pp. 92-104, 2017.

[27] H. Li, Z. Lu, J. Wu, H. Yu, X. Yu, and R. Chen, "Hydrothermal synthesis of transition metal oxide nanomaterials in HEPES buffer solution," Materials Letters, vol. 64, no. 18, pp. 1939-1942, 2010.

[28] G. Maiorano, L. Rizzello, M. A. Malvindi et al., "Monodispersed and size-controlled multibranched gold nanoparticles with nanoscale tuning of surface morphology," Nanoscale, vol. 3, no. 5, pp. 2227-2232, 2011.

[29] G. Plascencia-Villa, D. Bahena, A. R. Rodríguez, A. Ponce, and M. José-Yacamán, "Advanced microscopy of star-shaped gold nanoparticles and their adsorption-uptake by macrophages," Metallomics, vol. 5, no. 3, pp. 29-32, 2013.

[30] F. Tian, J. Conde, C. Bao, Y. Chen, J. Curtin, and D. Cui, "Gold nanostars for efficient in vitro and in vivo real-time SERS detection and drug delivery via plasmonic-tunable Raman/FTIR imaging," Biomaterials, vol. 106, pp. 87-97, 2016.

[31] K. R. Brown and M. J. Natan, "Hydroxylamine seeding of colloidal Au nanoparticles in solution and on surfaces," Langmuir, vol. 14, no. 4, pp. 726-728, 1998.

[32] L. Jiang, Y. Tang, C. Liow et al., "Synthesis of fivefold stellate polyhedral gold nanoparticles with 110 -facets via a seed-mediated growth method," Small, vol. 9, no. 5, pp. 705-710, 2013.

[33] K. Ito, K. Furuya, Y. Okano, and L. Hamada, "Development and Characteristics of a Biological Tissue-Equivalent Phantom for Microwaves," Electron. Commun. Japan Part I Commun, vol. 84, pp. 67-77, 2001.

[34] S. K. Sivaraman, S. Kumar, and V. Santhanam, "Monodisperse sub-10nm gold nanoparticles by reversing the order of addition in Turkevich method - The role of chloroauric acid," Journal of Colloid and Interface Science, vol. 361, no. 2, pp. 543-547, 2011.

[35] M. Zhu, B. Lei, F. Ren et al., "Branched Au Nanostructures enriched with a uniform facet: facile synthesis and catalytic performances," Scientific Reports, vol. 4, no. 1, article 05259, 2015.

[36] W. Ahmed, E. Stefan Kooij, A. Van Silfhout, and B. Poelsema, "Controlling the morphology of multi-branched gold nanoparticles," Nanotechnology, vol. 21, no. 12, Article ID 125605, 2010.

[37] P. Senthil Kumar, I. Pastoriza-Santos, B. Rodríguez-González, F. Javier García De Abajo, and L. M. Liz-Marzán, "High-yield synthesis and optical response of gold nanostars," Nanotechnology, vol. 19, no. 1, Article ID 015606, 2008.

[38] L. Zhao, X. Ji, X. Sun, J. Li, W. Yang, and X. Peng, "Formation and stability of gold nanoflowers by the seeding approach: the effect of intraparticle ripening," The Journal of Physical Chemistry C, vol. 113, no. 38, pp. 16645-16651, 2009.
[39] D. H. M. Dam, J. H. Lee, P. N. Sisco et al., "Direct observation of nanoparticle-cancer cell nucleus interactions," ACS Nano, vol. 6, no. 4, pp. 3318-3326, 2012.

[40] H. Yuan, M. Wanhong, C. Chen et al., "Shape and SPR evolution of thorny gold nanoparticles promoted by silver ions," Chemistry of Materials, vol. 19, no. 7, pp. 1592-1600, 2007.

[41] O. Bibikova, A. Popov, A. Bykov et al., "Plasmon-resonant gold nanostars with variable size as contrast agents for imaging applications," IEEE Journal of Selected Topics in Quantum Electronics, vol. 22, no. 3, Article ID 2526602, pp. 13-20, 2016.

[42] L. Minati, F. Benetti, A. Chiappini, and G. Speranza, "One-step synthesis of star-shaped gold nanoparticles," Colloids and Surfaces A: Physicochemical and Engineering Aspects, vol. 441, pp. 623-628, 2014.

[43] A. Gole and C. J. Murphy, "Seed-mediated synthesis of gold nanorods: role of the size and nature of the seed," Chemistry of Materials, vol. 16, no. 19, pp. 3633-3640, 2004.

[44] R. G. Weiner and S. E. Skrabalak, "Metal dendrimers: Synthesis of hierarchically stellated nanocrystals by sequential seedDirected overgrowth," Angewandte Chemie International Edition, vol. 54, no. 4, pp. 1181-1184, 2015.

[45] M. S. Bakshi, "How surfactants control crystal growth of nanomaterials," Crystal Growth and Design, vol. 16, no. 2, pp. 11041133, 2016.

[46] A. Zoppi, S. Trigari, G. Margheri, M. Muniz-Miranda, and E. Giorgetti, "Gold nanostars as SERS-active substrates for FTRaman spectroscopy," RSC Advances, vol. 5, no. 11, pp. 85238532, 2015.

[47] B. F. Jones, "A reappraisal of the use of infrared thermal image analysis in medicine," IEEE Transactions on Medical Imaging, vol. 17, no. 6, pp. 1019-1027, 1998.

[48] J. Teich, "Digital infrared imaging for medicine. Recent advances in I.R. focal plane array imaging technology," in Proceedings of the 18th Annual International Conference of the IEEE Engineering in Medicine and Biology Society, pp. 2079-2080, Amsterdam, Netherlands.

[49] J. P. Gore and L. X. Xu, “Thermal imaging for biomedical and medical diagnostics," in Biomedical Photonics Handbook, T. VoDinh, Ed., CRC Press, Boca Raton, FL, USA, 2003.

[50] C. Farcau and S. Astilean, "Mapping the SERS efficiency and hot-spots localization on gold film over nanospheres substrates," The Journal of Physical Chemistry C, vol. 114, no. 27, pp. 11717-11722, 2010.

[51] A. J. Pasquale, B. M. Reinhard, and L. Dal Negro, "Engineering photonic-plasmonic coupling in metal nanoparticle necklaces," ACS Nano, vol. 5, no. 8, pp. 6578-6585, 2011.

[52] X. Zhu, W. Feng, J. Chang et al., “Temperature-feedback upconversion nanocomposite for accurate photothermal therapy at facile temperature," Nature Communications, vol. 7, Article ID 10437, 2016.

[53] G. Chirico, P. Pallavicini, and M. Collini, "Gold nanostars for superficial diseases: A promising tool for localized hyperthermia?" Nanomedicine, vol. 9, no. 1, pp. 1-3, 2014. 


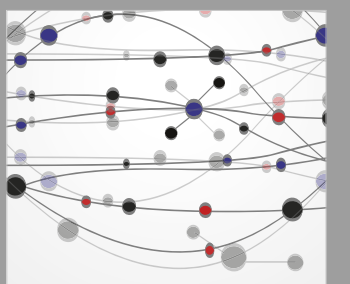

The Scientific World Journal
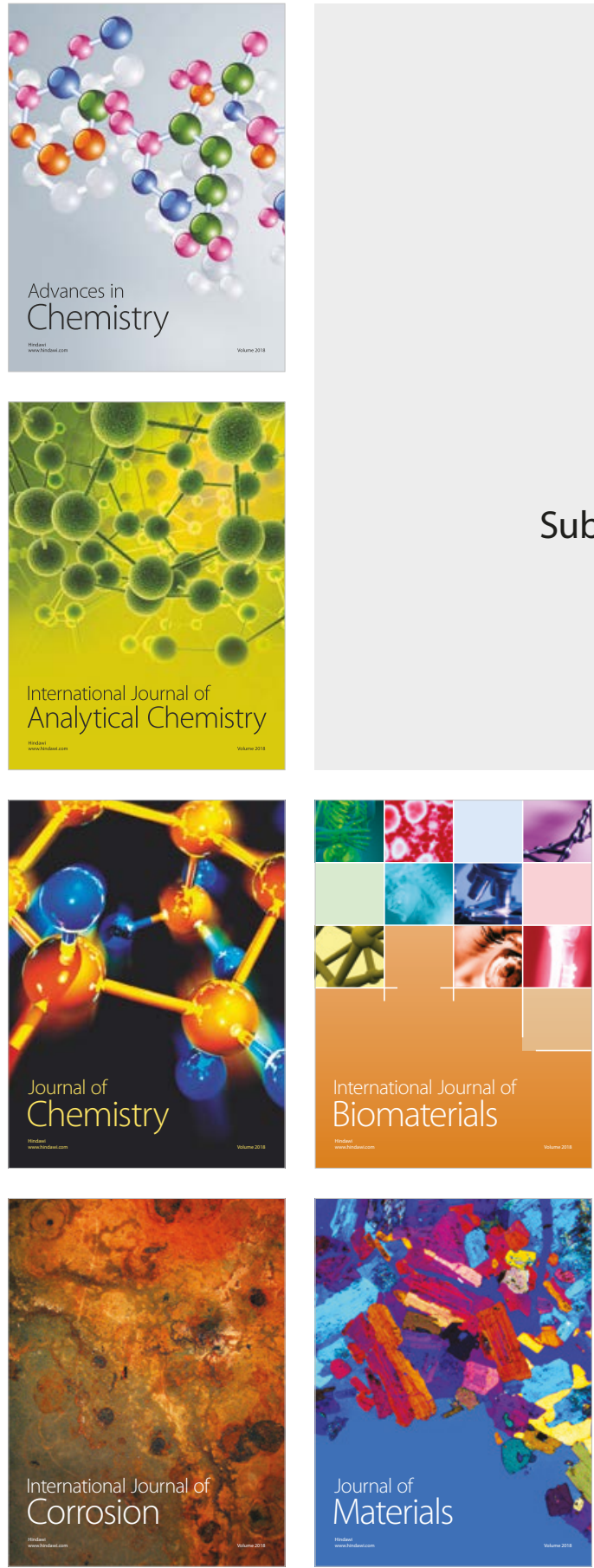

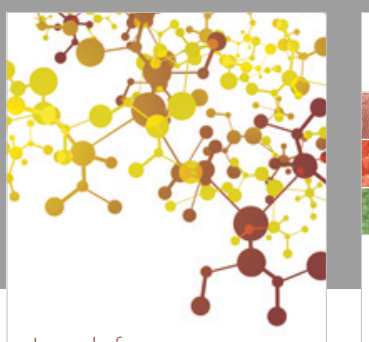

Journal of

Applied Chemistry
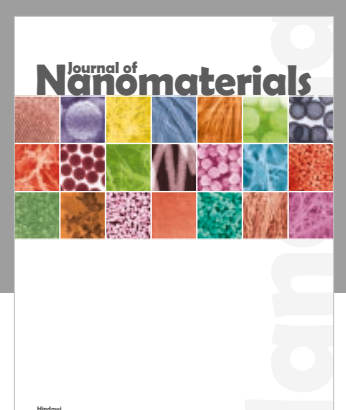

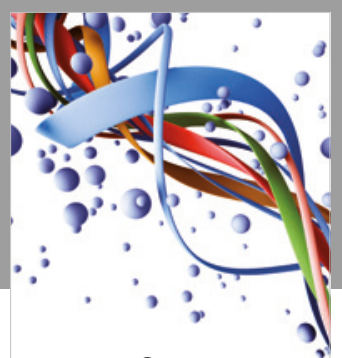

Scientifica

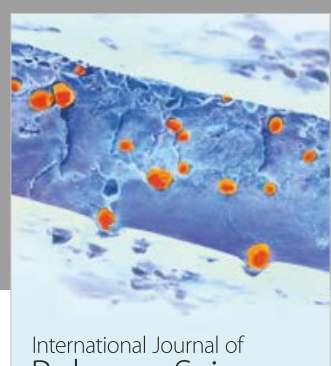

Polymer Science

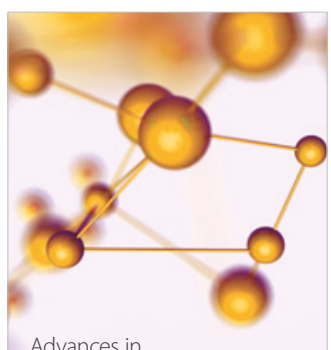

Physical Chemistry
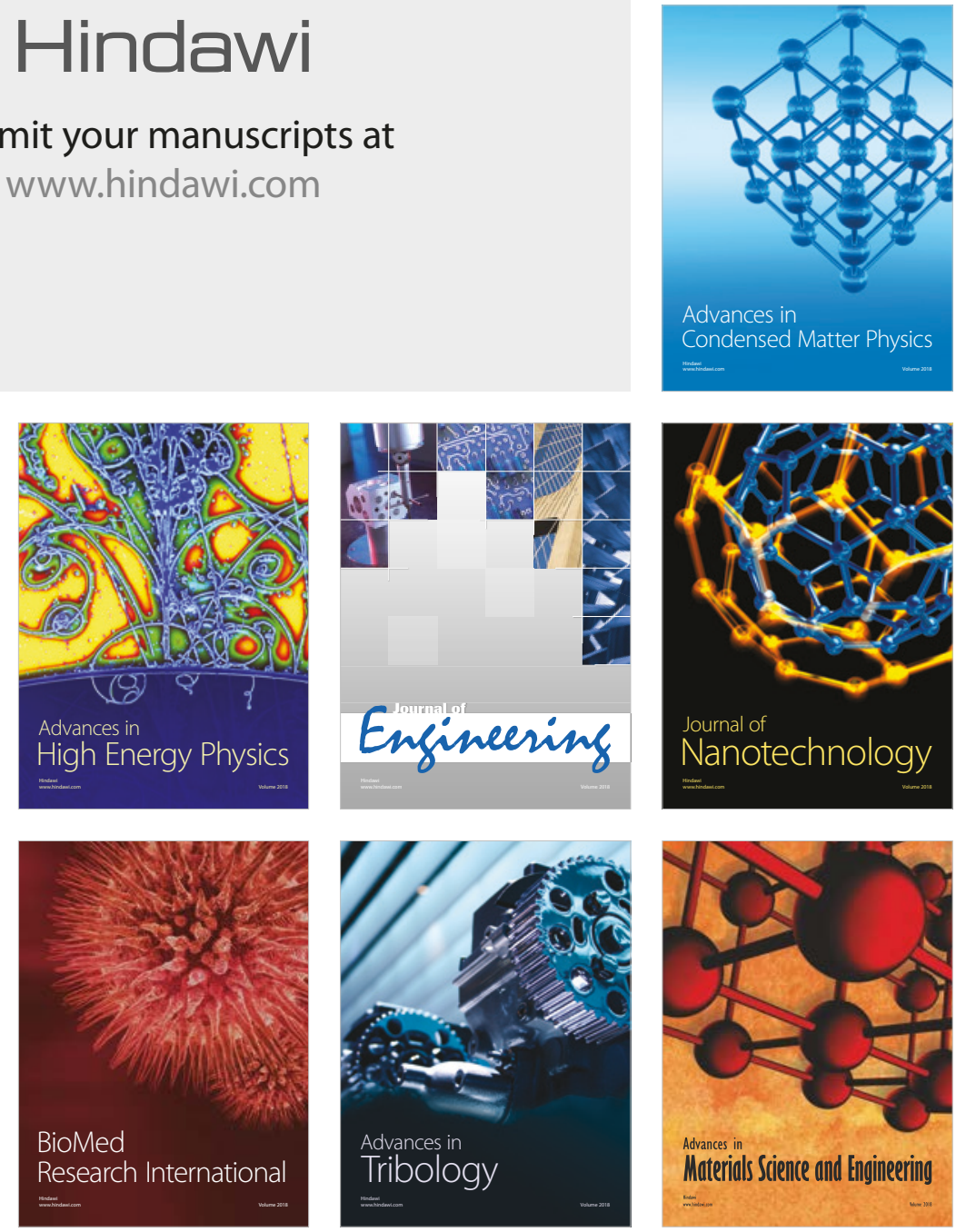